\title{
Colocación de catéter peridural en el paciente de dolor y cuidado paliativo: revisión narrativa
}

\section{Epidural catheter placement in the patient with pain and palliative care: Narrative Review}

\author{
María Margarita Viteri-Toro \\ Pontificia Universidad Javeriana, Colombia \\ María Camila Viteri-Toro \\ Pontificia Universidad Javeriana, Colombia \\ Diego Alberto Moreno-Martínez ${ }^{a}$ \\ Hospital Universitario San Ignacio, Colombia
}

\footnotetext{
a Autor de correspondencia. Correo electrónico: diegomoreno@javeriana.edu.co

Cómo citar: Viteri-Toro MM, Viteri-Toro MC, Moreno-Martínez DA. Colocación de catéter peridural en el paciente de dolor y cuidado paliativo: revisión narrativa. Univ Med. 2017;58(4):1-8. doi: https://doi.org/10.11144/Javeriana.umed58-4.ccpp
}

\section{RESUMEN}

El $10 \%$ de los pacientes con dolor oncológico que se encuentra con terapia sistémica farmacológica óptima presenta inadecuado control, explicado por refractariedad al tratamiento o efectos adversos intolerables. A pesar de que las técnicas intervencionistas se consideren el cuarto escalón de la escalera analgésica propuesta por la Organización Mundial de la Salud, en pacientes con cáncer, la aplicación anticipada de métodos intervencionistas (analgesia neuroaxial o de nervios periféricos) puede representar ventajas como un adecuado control del dolor. Se sugiere un enfoque multidisciplinario que incluya varios servicios clínicos para generar el mayor impacto de este tipo de técnicas en el tratamiento del dolor oncológico, teniendo en cuenta sus indicaciones y contraindicaciones. La analgesia por vía neuroaxial, dirigida directamente a las vías de transmisión del dolor en la médula espinal, proporciona mejor control del dolor, permite la reducción de las dosis de analgésicos y mejora la calidad de vida de los pacientes. La elección entre sistemas de administración de fármacos, ya sea de forma implantable y bombas externas, depende principalmente de expectativa de vida del paciente.

\section{Palabras clave}

dolor; dolor crónico; manejo; analgesia; neoplasia.

\begin{abstract}
$10 \%$ of patients with cancer pain who are on optimal pharmacological systemic therapy have inadequate control, explained by treatment refractoriness or intolerable adverse effects. Although interventional techniques are considered as the fourth step of the pain management ladder proposed by the World Health Organization, in cancer patients, the anticipated application of these interventional methods (such as neuroaxial analgesia and nerve blocks) can represent advantages. It is suggested a multidisciplinary approach that includes several clinical services to generate the greatest impact of this type of techniques in the treatment of cancer pain, taking into account its indications and contraindications. The analgesia via the neuroaxial route, directly directed to the spinal cord pain transmission routes, provides better
\end{abstract}


control of pain, allows the reduction of analgesic doses and improves the quality of life of the patients. The choice between drug delivery systems, either implantable and external pumps depends mainly on the life expectancy of the patient

Keywords

pain; chronic pain; pain management; analgesia; neoplasm.

\section{Introducción}

El manejo del dolor oncológico puede ser refractario al tratamiento farmacológico sistémico o presentarse efectos secundarios intolerables que perturban gravemente la calidad de vida de los pacientes [1]. El $10 \%$ de los pacientes que cuenta con terapia sistémica farmacológica óptima evidencia un inadecuado control del dolor [2].

Las intervenciones terapéuticas, como los bloqueos nerviosos y la analgesia neuroaxial (intratecal o epidural), permite que aquellos pacientes refractarios a todas las estrategias convencionales o limitados por los efectos secundarios alcancen un adecuado control del dolor. Se ha demostrado mejor control del dolor y en el perfil de los efectos adversos cuando se adicionan técnicas intervencionistas [3].

La aplicación adecuada de analgesia por vía neuroaxial proporciona mejor control del dolor, permite la reducción de dosis de analgésicos y mejora la calidad de vida de los pacientes [1], aunque este tipo de técnicas presenten una mayor complejidad en la atención y un riesgo de complicaciones poco frecuentes, pero potencialmente graves [2].

Estas técnicas se consideran el cuarto escalón de la escalera analgésica propuesta por la Organización Mundial de la Salud [2, 4, 5]; sin embargo, en pacientes con cáncer, la aplicación anticipada de estos métodos intervencionistas puede representar ventajas [1]. La administración epidural e intratecal de analgésicos se dirige directamente a las vías de transmisión del dolor en la médula espinal [1]. Su colocación es posible por puertos externos o internos; con sistemas de administración fijos o programables [3].

\section{Nivel de evidencia y grado de recomendación}

Los niveles de evidencia y grados de recomendación se basan en el Canadian Task Force on Preventive Heath Care [6] (tablas 1 y 2).

Tabla 1.Grados de recomendación para intervenciones de prevención

\begin{tabular}{|c|l|}
\hline $\begin{array}{c}\text { Grado de } \\
\text { recomendación }\end{array}$ & Interpretación \\
\hline $\mathrm{A}$ & $\begin{array}{l}\text { Existe buena evidencia para recomendar la } \\
\text { intervención clínica. }\end{array}$ \\
\hline $\mathrm{B}$ & $\begin{array}{l}\text { Existe moderada evidencia para recomendar la } \\
\text { intervención clínica. }\end{array}$ \\
\hline $\mathrm{C}$ & $\begin{array}{l}\text { La evidencia disponible es conflictiva y no permite } \\
\text { hacer recomendaciones a favor o en contra de la } \\
\text { intervención clínica; sin embargo, otros factores } \\
\text { podrían influenciar la decisión. }\end{array}$ \\
\hline $\mathrm{D}$ & $\begin{array}{l}\text { Existe moderada evidencia para recomendar en } \\
\text { contra la intervención clínica. }\end{array}$ \\
\hline $\mathrm{E}$ & $\begin{array}{l}\text { Existe buena evidencia para recomendar en contra } \\
\text { la intervención clínica. }\end{array}$ \\
\hline $\mathrm{I}$ & $\begin{array}{l}\text { Existe evidencia insuficiente (en calidad y cantidad) } \\
\text { para hacer una recomendación; sin embargo, otros } \\
\text { factores podrín influenciar la decisión. }\end{array}$ \\
\hline
\end{tabular}

Tabla 2.Niveles de evidencia e interpretación de los tipos de estudios

\begin{tabular}{|c|l|}
\hline $\begin{array}{c}\text { Niveles de } \\
\text { evidencia }\end{array}$ & Interpretación \\
\hline I & $\begin{array}{l}\text { Evidencian a partir de ensayos clínicos con } \\
\text { asignación aleatoria. }\end{array}$ \\
\hline II-1 & $\begin{array}{l}\text { Evidencian a partir de ensayos clínicos sin asignación } \\
\text { aleatoria. }\end{array}$ \\
\hline II-2 & $\begin{array}{l}\text { Evidencia a partir de estudios de cohortes y casos y } \\
\text { controles, preferiblemente realizados por más de un } \\
\text { centro o un grupo de investigación. }\end{array}$ \\
\hline II-3 & $\begin{array}{l}\text { Evidencia a partir de comparaciones en el tiempo o } \\
\text { en sitios, con la intervención o sin esta; podrían } \\
\text { incluirse resultados provenientes de estudios sin } \\
\text { asignación aleatoria. }\end{array}$ \\
\hline $\begin{array}{l}\text { Opinión de expertos, basados en la experiencia } \\
\text { clínica; estudios descriptivos o informes de comités } \\
\text { de expertos. }\end{array}$ \\
\hline
\end{tabular}

\section{Propósito}

El uso de esta técnica intervencionista se propone principalmente en situaciones donde analgésicos orales, transdérmicos o endovenosos tienen un efecto insuficiente o producen efectos secundarios intolerables; por lo tanto, la 
administración central de los analgésicos está indicada para aumentar el efecto analgésico y reducir el riesgo de efectos secundarios y, de esta forma, mejorar la calidad de vida de los pacientes [1]. Se conoce que la analgesia epidural continua es efectiva entre el $70 \%$ y el $90 \%$ de los pacientes, con mejoría del dolor en el $72 \%$ de pacientes con dolor oncológico [5].

\section{Normas del procedimiento}

Debe existir un enfoque multidisciplinario que incluya los servicios:

Oncología clínica.

Servicios quirúrgicos relacionados.

Radioterapia.

Psicología.

Psiquiatría

Servicio de dolor oncológico-cuidado paliativo.

Servicio de intervencionismo analgésico.

Otros servicios.

Este enfoque optimiza el impacto de este tipo de técnicas como parte del tratamiento oncológico [2].

\section{Preparación}

La preparación del paciente para la analgesia epidural se constituye por:

1. Proceso de selección del paciente.

2. Firma del consentimiento informado.

3. Equipo de monitorización y reanimación.

4. Obtención de un acceso intravenoso.

5. Selección de los medicamentos apropiados, dependiendo de las enfermedades coexistentes del paciente.

La técnica debe ser estrictamente estéril, debido a que a menudo se deja un catéter in situ [7].

\section{Equipo}

Se han utilizado varios tipos de agujas epidurales, pero las agujas de Tuohy son las más usadas
[7]. Generalmente, son de calibre 16-18 Gauge y tienen una punta roma "Huber" curvada de $15^{\circ}-30^{\circ}$, diseñada para reducir el riesgo de punción accidental de la duramadre y guiar el catéter. El cuerpo de la aguja está marcado en intervalos de $1 \mathrm{~cm}$, de manera que puede identificarse la profundidad de la inserción [7].

El catéter está hecho de un plástico calibrado, flexible, duradero, con un solo orificio al final o con múltiples orificios laterales cerca de la punta para la dispersión del medicamento [7] (figuras 1 y 2).

Figura 1.Equipo del catéter peridural (izq.) y aguja de Tuohy (der.)
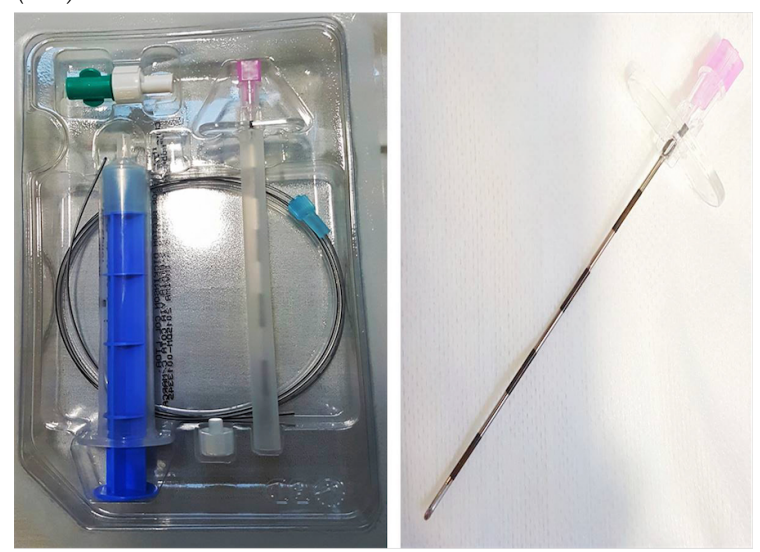

Figura 2.Punta de la aguja de Tuohy

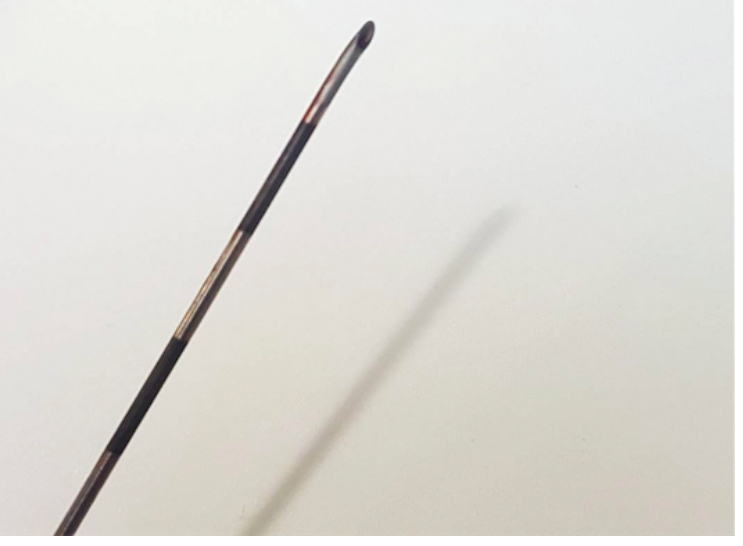

\section{Posición}

Las posiciones que puede asumir el paciente para la inserción del catéter peridural son [7]: 
Decúbito lateral.

Sentado: se utiliza principalmente en pacientes obesos ya que ayuda a la identificación de la línea media [8].

Decúbito prono.

\section{Nivel de punción}

La zona de inserción de la aguja depende del área de analgesia que se quiera lograr. Entre los puntos de referencia superficiales importantes están [7]:

La línea de Tuffier o intercrestal: correspondiente al espacio intervetebral L4-L5.

El ángulo inferior de la escápula: correspondiente al cuerpo vertebral T7.

La raíz de la espina escapular: correspondiente al cuerpo vertebral T3.

Vértebra prominente: en la unión cérvicotorácica correspondiente al cuerpo vertebral C7.

Algunos datos sugieren que una estimación clínica de un espacio intervertebral basado en la anatomía de la superficie puede no ser precisa en muchos pacientes $y$, a menudo, resulta en la colocación de la aguja uno o dos niveles de la columna vertebral diferente al previsto [9]. Así mismo, la línea de Tuffier no es un punto de referencia constante [10]. Por lo tanto, la necesidad de establecer un método más fiable para identificar los niveles de la columna vertebral y el avance de la aguja hacia el neuroeje sigue inspirando estudios clínicos e imagenológicos. La introducción del ultrasonido en la anestesia regional ha permitido representar la ubicación y relación espacial (por ejemplo, profundidad) de los puntos de referencia óseos para ayudar a estimar tanto el sitio correcto de la punción, así como la profundidad de inserción de la aguja [11].

\section{Abordajes}

Existen distintos abordajes para la inserción del catéter peridural [7]:

Línea media o medial: es el abordaje más utilizado; es elegido habitualmente para los accesos lumbar y torácico bajo [7].
Paramedial: es particularmente útil en la región torácica media o alta, donde la inclinación de la columna vertebral y los espacios estrechos convierten en problemático el acceso por línea media.

Abordaje de Taylor: es un acceso paramedial modificado a través del interespacio L5-S1, que puede ser útil en pacientes con traumatismos que no toleran o no son capaces de mantener una posición sentada.

Caudal

\section{Dirección de aguja}

El ángulo de acceso debe ser ligeramente cefálico $\left(10^{\circ}-15^{\circ}\right)$ en las regiones lumbar y torácica baja; mientras que en la región torácica media el enfoque deberá ser más cefálico, debido a la mayor inclinación hacia caudal de las apófisis espinosas en esta región de la columna [7].

\section{Punción (abordaje por línea media)}

1. Preparar la piel con una solución antiséptica (la Sociedad Americana de Anestesia Regional actualmente recomienda soluciones cloradas o alcoholadas para la antisepsia de los procedimientos de anestesia regional) [8].

2. Infiltrar la piel y el tejido celular subcutáneo que será el trayecto de la aguja con anestésico local hasta una profundidad variable en función del hábito corporal, con el fin de disminuir el dolor en la inserción de la aguja y, así mismo, ayuda a reconocer el trayecto correcto para la aguja [8].

3. Tomar la aguja con los dedos índices y pulgar de la mano dominante sosteniendo el eje o el ala de la aguja. Insertar la aguja en la mitad del espacio intervertebral elegido y de forma controlada la aguja debe ser avanzada con el estilete a través de la piel, tejido celular subcutáneo, ligamento supraespinoso y el ligamento 
interespinoso, momento en el que el estilete puede ser retirado y la jeringa de pérdida de resistencia unida. Si está en la ubicación correcta, la aguja debe descansar firmemente en los tejidos $[7,8]$.

4. Cuando la aguja se ubica en el ligamento interespinoso, la aguja se avanza lentamente a través de este hasta que se note un aumento en la resistencia. Este aumento de la resistencia representa el ligamento amarillo [8]. Por lo general, el ligamento amarillo se identifica como una estructura más dura con aumento de la resistencia y cuando se entra posteriormente en el espacio epidural, la presión aplicada al émbolo de la jeringa permite que la solución salina o el aire fluyan sin resistencia dentro del espacio epidural [7].

\section{Identificación del espacio epidural}

El método de identificación del espacio peridural debe ser predefinido [7]. Existen diferentes métodos para la identificación del espacio epidural, entre los que encontramos:

Pérdida de resistencia. El método más usado es la técnica de pérdida de resistencia al paso de la duramadre con aire o con solución salina [12]. Cada uno implica una suave presión intermitente (para el aire) o constante (para la solución salina) aplicada sobre el émbolo de la jeringa de pérdida de resistencia; mientras que se avanza la aguja por el ligamento interespinoso hasta pasar por el ligamento amarillo [7]. Una desventaja potencial de esta técnica es su baja especificidad, en muchos casos debido a quistes de los ligamentos interespinosos (en la región lumbar se presentan hasta en el $85 \%$ en las personas entre 61 y 79 años de edad), así como la ubicación de la aguja entre los músculos paravertebrales y las brechas del ligamento amarillo en la línea media, los cuales pueden resultar en un falso positivo de pérdida de resistencia desde un $8,3 \%$ hasta un 17 $\%$ de los pacientes [12].
Reconocimiento de presión negativa o prueba de gota pendiente: después de colocar la aguja dentro del ligamento amarillo, se instila una gota de solución salina en el interior del hueco de la aguja. Si la aguja está en el espacio epidural, la solución debe ser succionada [7,12].

Caída acústica del tono [12].

Debido al alto porcentaje de falsos positivos en la pérdida de resistencia, se han propuesto varios métodos para la confirmación de la adecuada ubicación en el espacio epidural:

Imágenes radiográficas: la guía fluoroscópica ofrece muchas ventajas para la inserción del catéter epidural. Además de guiar la aguja entre las apófisis espinosas o entre las láminas, permite la confirmación de la pérdida de resistencia obtenida por la aguja epidural, así como la colocación del catéter dentro del espacio epidural y descarta su posicionamiento en el espacio intratecal e intravascular.

Dosis de prueba: la dosis de prueba epidural se utiliza para descartar inyección intratecal e intravascular de los anestésicos por mal posicionamiento del catéter. Se utiliza tradicionalmente para este propósito un pequeño volumen de lidocaína al 1,5\% con adrenalina, usando la referencia de un aumento de la presión arterial sistólica mayor de $15 \mathrm{mmHg}$ o un aumento en la frecuencia cardiaca mayor de 10 latidos/min [7].

Punción dural: cuando se insertan catéteres epidurales lumbares por debajo del cono medular (L1-L2), una aguja espinal puede ser introducida a través de la aguja epidural para llevar a cabo técnica combinada espinal-epidural. La punción dural también proporciona confirmación de la pérdida de resistencia: si se obtiene retorno de líquido cefalorraquídeo a través de la aguja espinal, la punta de la aguja epidural debe estar más probablemente colocada en el espacio epidural.

Análisis de forma de onda epidural: trata de medir el cambio de presión de la aguja (la cual está conectada a un transductor de presión) al pasar por el ligamento amarillo. La penetración en el espacio epidural resulta en una caída repentina de presión de los tejidos y el 
trazado de la presión se vuelve sincrónico con las pulsaciones arteriales.

Ultrasonografía: hay dos formas para ayudar a la correcta inserción del catéter peridural por medio de la ecografía: exploración de la anatomía previo a la inserción y orientación en tiempo real, la cual intenta visualizar el avance ecográfico de la aguja y su penetración al espacio epidural [12].

\section{Inserción del catéter}

1. Cuando se identifica el espacio epidural, debe anotarse la profundidad de la aguja desde la piel hasta el espacio epidural.

2. Se inserta suavemente el catéter mediante la aguja hasta aproximadamente la marca de $15-18 \mathrm{~cm}$, para asegurar que una longitud suficiente ha entrado en el espacio epidural.

3. Debe retirarse cuidadosamente la aguja, posterior a lo cual se retira el catéter hasta dejar 4-6 cm en el espacio epidural. Un asiento del catéter menor de $4 \mathrm{~cm}$ de longitud en el espacio epidural puede aumentar el riesgo de desprendimiento del catéter y de analgesia inadecuada; mientras que insertar más catéter puede aumentar la probabilidad de una posición defectuosa o complicaciones relacionadas [7].

\section{Indicaciones}

Las indicaciones para cambiar el tratamiento analgésico sistémico a analgesia epidural e intradural son $[1,2]$ :

Resistencia a altas dosis de opioides orales, transdérmicos o sistémicos.

Dolor que es sensible a los opioides sistémicos, pero acompañado de efectos secundarios intolerables como náuseas, vómitos, estreñimiento o reacciones alérgicas.

Dolor que no puede ser tratado con otras modalidades intervencionistas.

La vía de administración epidural está indicada como tratamiento analgésico de etiología focalizada y cuando el periodo de tratamiento es corto [1].

Otra indicación de analgesia epidural es en pacientes con supervivencia prevista inferior a tres meses [5]. La elección entre sistemas de administración de fármacos, ya sea de forma implantable y bombas externas, depende principalmente de expectativa de vida del paciente $[1,13]$.

\section{Contraindicaciones}

Las contraindicaciones para el uso de analgesia epidural e intradural son [1]:

1. Presión intracraneal elevada (considerada una contraindicación absoluta) [2].

2. Infecciones generalizada o localizada en el sitio de inserción.

3. Masa tumoral sospechosa o confirmada en el sitio de inserción.

4. Diátesis hemorrágicas.

5. Reacción alérgica a los medicamentos que se utilizarán en el espacio epidural o intratecal.

6. Agitación o trastornos cognitivos que pueden inducir el retiro del catéter de forma inesperada.

El uso crónico de anticoagulantes no es una contraindicación para las técnicas neuroaxiales. Se debe realizar el manejo pertinente de la anticoagulación previo a la realización de dichas técnicas según las guías de la Asociación Americana de Anestesia Regional [2].

Además, se considera que los hallazgos imagenológicos de metástasis cerebrales aumentan significativamente el riesgo de complicaciones (herniación y hemorragia), por lo que también podría considerarse una contraindicación [2].

\section{Complicaciones}

Las infecciones son el mayor problema asociado al uso de catéteres neuroaxiales [5]. Un 
estudio encontró que las infecciones ocurren en un $1 \%$ en la técnica epidural y en un $2 \%$ en la intratecal. En los pacientes con catéter epidural, el $16 \%$ requerirían el cambio o su retiro por complicaciones asociadas [1]. Las infecciones se desarrollan más rápidamente con catéteres con largo tiempo de inserción [5].

Una revisión sistemática y metanálisis investigó las tasas de infección asociadas a catéteres epidurales durante por lo menos 7 días. Los resultados revelan que el 6,1\% de las infecciones se relacionaban con el catéter, el 4,6 $\%$ de infecciones eran superficiales y el 1,2\% eran profundas. La incidencia de las infecciones relacionadas con el catéter se calculó en 0,4/000 días de catéter [14].

Otras complicaciones relacionadas con el catéter, como la obstrucción mecánica, se han reportado con diferentes tasas de incidencia entre el $1 \%$ y el $44 \%$ [1].

Se cree que los analgésicos por vía neuroaxial presentan menor toxicidad sistémica que por vía oral o intravenosa [2,15]. Las dosis altas de anestésicos locales por vía intratecal o espinal pueden producir debilidad motora y deficiencias sensoriales, además de retención urinaria e hipotensión arterial transitoria [1].

No hay informes en la literatura acerca de los efectos tóxicos sobre el sistema nervioso en caso de administración prolongada de anestésicos locales en el neuroeje [1].

\section{Riesgos}

Los riesgos asociados al equipo utilizado para el implante de catéteres epidurales son [2]:

Lesión nerviosa o parálisis: debida a daño directo de la médula espinal con la aguja, sangrado o compresión por hematoma epidural o a través del gradiente de presión en el líquido cefalorraquídeo, que causa impactación de la médula contra la duramadre [16].

Cefalea pospunción dural.

Formación de granulomas.

El riesgo de fibrosis en la punta del catéter es más común con infusiones epidurales, sobre todo en aquellas prolongadas [5], lo que da lugar a fracaso del tratamiento o aumento del dolor secundario a la acumulación del medicamento en un espacio cerrado creado por la fibrosis.

\section{Recomendaciones}

Las técnicas de administración epidural de analgésicos supervisadas por un equipo multidisciplinario deben ser incluidas como parte de la estrategia de tratamiento del dolor oncológico (II-B) [2].

\section{Referencias}

1. Vissers KC, Besse K, Wagemans $\mathrm{M}$, Zuurmond W, Giezeman MJ, Lataster A, et al. Evidence-based interventional pain medicine. 23. Pain in patients with cancer. Pain Pr. 2011;11:453-75.

2. Smyth CE, Jarvis V, Poulin P. Brief review: Neuraxial analgesia in refractory malignant pain. Can J Anesth. 2014;61(2):141-53.

3. Jost L, Roila F. Management of cancer pain: ESMO Clinical Practice Guidelines. Ann Oncol. 2010;21 Suppl 5:v257-60.

4. Vargas-Schaffer G. Is the WHO analgesic ladder still valid?: Twenty-four years of experience. Can Fam Physician. 2010;56(6):514-7.

5. Jeon YS, Lee JA, Choi JW, Kang EG, Jung HS, Kim HK, et al. Efficacy of epidural analgesia in patients with cancer pain: a retrospective observational study. Yonsei Med J. 2012;53(3):649-53.

6. Mella S M, Zamora P, Mella L M, Alfaro J, Uceda P. Niveles de evidencia clínica y grados de recomendación. Rev Soc Andaluza Trauma y Ortop. 2012;29(1-2):59-72.

7. Brown DL, Wedel DJ, Miller RD. Anestesia intradural, epidural y caudal. En: Miller RD, editor. Anestesia. Barcelona: Doyma; 1993. p. 1269-74.

8. Paul G, Bruce F, Robert K, Bernards CM. Chapter 25: Epidural and spinal anesthesia. Clinical Anesthesia. 2006. 1-86 p. 
9. Teoh DA, Santosham KL, Lydell CC, Smith DF, Beriault MT. Surface anatomy as a guide to vertebral level for thoracic epidural placement. Anesth Analg. 2009;108(5):1705-7.

10. Reynolds F. Damage to the conus medullaris following spinal anaesthesia. Anaesthesia. 2001 Mar;56(3):238-47.

11. Manoj K, Vandepitte C. Introduction to ultrasound guided neuraxial and perineuraxial techniques. NYSORA [Internet]. 2013. Disponible en: http://ww w.nysora.com/techniques/neuraxial-and-p erineuraxial-techniques/ultrasound-guided /3275-introduction.html

12. Tran DQH, González AP, Bernucci F, Finlayson RJ. Confirmation of loss-ofresistance for epidural analgesia. Reg Anesth Pain Med. 2015;40(2):166-73.

13. Stearns L, Boortz-Marx R, Du Pen S, Friehs G, Gordon M, Halyard M, et al. Intrathecal drug delivery for the management of cancer pain: a multidisciplinary consensus of best clinical practices. J Support Oncol. 2005;3 (6):399-408.

14. Ruppen W, Derry S, McQuay HJ, Moore $\mathrm{RA}$. Infection rates associated with epidural indwelling catheters for seven days or longer: systematic review and metaanalysis. BMC Palliat Care. 2007;6:3.

15. Mercadante S, Porzio G, Gebbia V. Spinal analgesia for advanced cancer patients: An update. Crit Rev Oncol/Hematol. 2011;82:227-32.

16. Chai T, Bruel BM, Nouri KH, Driver L. Complications after intrathecal drug delivery due to the underlying malignancy in two patients with intractable cancer pain. Pain Physician. 2013;16(2):E107-11. 